\title{
Hard cases of comparison
}

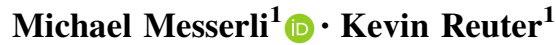

(C) Springer Science+Business Media Dordrecht 2016

\begin{abstract}
In hard cases of comparison, people are faced with two options neither of which is conceived of as better, worse, or equally good compared to the other. Most philosophers claim that hard cases (1) can indeed be distinguished from cases in which two options are equally good, and (2) can be characterized by a failure of transitive reasoning. It is a much more controversial matter and at the heart of an ongoing debate, whether the options in hard cases of comparison should be interpreted as incomparable, on par, or roughly equal. So far, however, none of these claims and interpretations have been tested. This paper presents the first empirical investigation on hard cases, intransitive reasoning, and incomparability. Our results reveal that hard cases present real-world dilemmas in which a significant majority of people violate transitivity. After suggesting a way of operationalizing the notion of incomparability, we provide empirical evidence that the options in some hard cases are not considered to be incomparable. Theories of rough equality or parity seem to provide better interpretations of our results.
\end{abstract}

Keywords Hard cases - Incomparability - Small-improvement argument · Transitivity $\cdot$ Intransitivity $\cdot$ Operationalization $\cdot$ Empirical studies

The authors' names are listed in alphabetic order. This work is fully collaborative.

Michael Messerli

michael.messerli@philo.unibe.ch

Kevin Reuter

kevin.reuter@philo.unibe.ch

1 Institute of Philosophy, University of Bern, Länggassstrasse 49a, 3000 Bern 9, Switzerland 


\section{Introduction}

Rational decision making can be difficult. Imagine you are confronted with a choice between having a successful career as a lawyer and having a successful career as an artist. ${ }^{1}$ While some people would surely have a clear preference for becoming either a lawyer or an artist, others might find this choice hard: having a successful career as a lawyer will be judged to be neither better than, nor worse than, nor equal to having a successful career as an artist. It also does not seem appropriate to solve the conflict on the basis of some randomizing procedure. ${ }^{2}$ This leads to the worrying conclusion that in such hard cases, it is not possible to decide rationally between the alternatives. To substantiate this claim, let us consider the following argument:

i. The possibility of rational choice between two options depends on them being comparable.

ii. Two options, $x$ and $y$, are comparable, if and only if $x$ is either better than, worse than, or equal to $y$.

iii. There are hard cases, in which $x$ is neither better, nor worse, nor equally good to $y$.

$\therefore$ There are hard cases, in which a rational decision cannot be made.

The argument is prima facie compelling: How can you decide rationally between a career as a lawyer and a career as an artist if a comparison cannot be made? How can you compare these options if one option is neither better than, nor worse than, nor equally good to another option? In this paper we focus on the second and the third premise: How shall we understand the phenomenon of a hard case? What is characteristic for it?

Some philosophers deny or are at least suspicious about the existence of hard cases. E.g. Regan (1997) endorses the thesis of strong comparability-the thesis that there is always a ranking of two options in terms of a common value. He writes: “(..) I am inclined to take comparability as my working hypothesis." (1997, p. 150). However, a majority of philosophers accept that hard cases of comparison exist. Chang (2002a, p. 671) and Boot (2007, p. 30), for instance, claim that we sometimes promote judgments that one option is neither better than, nor worse than, nor equally good to another option.

Not only is it part of the philosophical consensus that hard cases exist, it is generally accepted that such cases can be shown to violate transitivity. In order to show how transitivity fails in hard cases of comparison, we will consider the smallimprovement argument. The small-improvement argument is based upon the intuitive idea that in hard cases an option which is marginally improved $(\mathrm{x}+)$ is

\footnotetext{
1 This is a modified version of an example found in $\operatorname{Raz}$ (1986, p. 342).

2 In contrast, tossing a coin seems to be a reasonable strategy if the options are equally good. For a discussion on using a random process in hard case scenarios, see Schaber (1994, p. 158) and Chang (2002a, pp. 684-685; 2012, p. 118). Reuter and Messerli (2016) are in the process of investigating this issue empirically.
} 
always better than the original option (x) but not better than the alternative option (y). ${ }^{3}$ It can be easily illustrated with the example used above:

1. A career as a lawyer $(x)$ is neither better nor worse than a career as an artist $(y)$.

2. A career as a lawyer plus two additional holidays $(x+)$ is neither better nor worse than a career as an artist $(y)$.

3. A career as a lawyer plus two additional holidays $(x+)$ is better than a career as a lawyer $(x)$.

$\therefore$ A career as a lawyer is neither better than nor worse than nor equal to a career as an artist.

Anyone who endorses statements (1)-(3) reasons intransitively, because if transitivity were to hold, we could infer the negation of (3) from both (1) and (2). The small-improvement argument is, therefore, standardly considered to reveal a failure of transitivity (Boot 2007, p. 32). We can furthermore conclude from the three premises that in hard cases one option is neither better, nor worse, nor equal to another option-see Sect. 2.2. for a more detailed analysis of transitivity and the conclusion of the small-improvement argument. This conclusion is most commonly interpreted to show that in hard cases two options are incomparable. However, others have resisted such an interpretation of the small-improvement argument and instead suggested to conceive of options in hard cases as roughly comparable (Parfit 1986) or on par (Chang 2002a). While we agree that hard cases of comparison are particularly fascinating cases that raise important questions about violations of transitivity, the possibility of incomparability, as well as rationality, the existing literature is devoid of any empirical data that provides a foundation for the purely a priori discussions in the literature. More specifically, the debate has failed to address three important questions:

a. Are hard cases real-world dilemmas, i.e. do we really distinguish hard cases from easy cases of comparison?

b. Do people indeed violate transitivity in hard cases?

c. Can we operationalize the notion of incomparability and provide empirical evidence for or against that notion?

In order to answer these three questions, we will proceed as follows: Sect. 2 illustrates the various aspects of the small-improvement argument. Most importantly, we will discuss how to distinguish easy from hard cases, and how hard cases lead to a failure of transitive reasoning. We also propose a way to operationalize the notion of incomparability. In Sect. 3-the empirical part of this paper-we examine the empirical reality of hard cases, failure of transitivity, and incomparability. In order to investigate the a priori claims made by philosophers, we presented participants with both easy and hard case scenarios. People's responses were then

\footnotetext{
3 The argument is discussed in, e.g. De Sousa (1974), Raz (1986), Chang (2002a), Espinoza (2008), Boot (2009), Carlson (2011), Gustafsson (2013) and Andreou (2015).
} 
recorded to examine the response profiles in easy and hard cases of comparison. Section 4 concludes with a General Discussion combining the theoretical and the empirical part. In "Appendix 1" we analyze the role of (in)transitivity within the small-improvement argument. "Appendix 2" presents the vignettes of the empirical study.

\section{The small-improvement argument and its interpretations}

\subsection{The ingredients: hard cases of comparison}

We have introduced the small-improvement argument in the previous section. Let us now consider a general version of this argument:

1. $x$ is neither better nor worse than $y$.

2. $x+$ is neither better nor worse than $y$.

3. $x+$ is better than $x$.

$\therefore x$ is neither better than nor worse than nor equal to $y$.

In hard cases of comparison the first two premises seem to be true, e.g. lawyer versus artist. However, in easy cases of comparison the second premise does not hold. So-called easy cases of comparison are not situations in which it is easy to decide between two options. Instead, the options are likely to be considered equally good such that a small incentive will persuade the agent to choose the option that includes the small incentive $(x+)$. E.g., imagine you have the choice between a career at two equally appealing law firms. While a career as a lawyer at Smith and Partners $(x)$ is neither better nor worse than a career as a lawyer at Jones and Partners $(y)$, a career as a lawyer at Smith and Partners plus two additional holidays $(\mathrm{x}+)$ is ceteris paribus better than a career as a lawyer at Jones and Partners $(y)$.

Thus, we can see that the question of whether hard cases reveal a failure of transitivity does not need to be raised in order to differentiate easy from hard cases: different responses to the first two premises are sufficient to distinguish both kinds of choices. Not all philosophers do agree that hard cases differ from easy cases. Regan (1997) suggests that in all cases of comparison, either one option is better than the other or they are precisely equal in value (1997, p. 129). He will therefore reject the distinction between an easy and a hard case. Who is right, Regan or the philosophical consensus? The empirical studies we present in Sect. 3.1 were designed to address this issue. We will see that there is very little empirical support for Regan's position. Instead, the philosophical consensus seems to be largely confirmed.

\subsection{The mark of hard cases: failure of transitivity}

Whereas the first two premises of the small-improvement argument are sufficient to separate easy from hard cases, most philosophers have focused on a fascinating consequence of hard cases. In addition to the first two premises, any person who is 
willing to endorse premise (3) of the small-improvement argument, shows a failure of transitive reasoning. 4

To recognize the role of intransitivity within the small-improvement argument, let us first formalize each premise of the argument:

$$
\begin{array}{lll}
1 & \neg(x \succ y) \wedge \neg(y \succ x) & \text { premise } \\
2 & \neg(x+\succ y) \wedge \neg(y \succ x+) & \text { premise } \\
3 & x+\succ x & \text { premise }
\end{array}
$$

' $x$ ', ' $y$ ' and ' $x+$ ' represent the options that the agent is confronted with. ' $\succ$ ' means 'is better than'. ' $\prec$ ' means 'is worse than'. '=' means 'equal in value to'. ' $\neg$ ' stands for a negation. ' $\neg(x \succ y)$ ' for instance, says that it is not true that $x$ is better than $y$.

In the formalization above it can be seen that the small-improvement argument violates the following kind of transitivity:

$$
(x+\succ x \wedge \neg(y \succ x)) \rightarrow x+\succ y
$$

The conjunction of the three premises cannot be true, if the transitivity condition above is not violated. ${ }^{5}$

Intransitivity is therefore widely accepted as the mark of hard cases. However, no empirical evidence has been put forward to underline this claim. In Sect. 3.2 we show that a substantial majority of people indeed violate transitivity in hard cases.

\subsection{The interpretation: incomparability or rough comparability}

The small-improvement argument contains two premises (1) and (2) which allow us distinguish hard from easy cases. In conjunction with premise (3), we observe that violations of transitivity seem to be an unavoidable consequence of hard cases. In the last stage, we now need to consider the conclusion of the small-improvement argument. Recall that if $x$ is neither better nor worse than $y$, and $x+$ neither better nor worse than $y$, and $x+$ better than $x$, then we can conclude that

$\therefore x$ is neither better than nor worse than nor equal to $y$

\footnotetext{
${ }^{4}$ Raz states: "The test of incommensurability is failure of transitivity."(Raz 1986, p. 325). Hsieh (2005, p. 201) and Boot (2007, p. 32) are following Raz by claiming that failure of transitivity is characteristic for incomparability. Chang, recently interviewed, says that an important difference between equally good and on a par is that equally good is transitive, while on a par is not (Chang: 'The existentialist of hard choices'). For Parfit (1986), rough equality is the same as a non-transitive value relation (1986, p. 431).

5 It should be emphasized that the transitvity condition violated in these cases is not of the well known form such as $x$ is better than $y, y$ is better than $z$, and $x$ is better than $z$. Instead, it is logically equivalent with following negative transitivity principle:

$$
\neg(x+\succ y) \wedge \neg(y \succ x)) \rightarrow \neg(x+\succ x)
$$

For example, Bridges and Mehta discuss negative transitivity of the 'better than' relation (Bridges and Mehta 2013). We would like to stress that the role of (in)transitivity within the small-improvement argument is more complex. In "Appendix 1" we show two possibilities on how to move from the premises to the conclusion. These formalizations show that some versions of the small-improvement argument imply a different form of transitivity while others do not.
} 
But what does it mean for one option to be neither better than, nor worse than, nor equally good to another option? Many scholars have argued that the conclusion of the small-improvement argument means that both options are incomparable (De Sousa 1974, p. 546; Raz 1986, p. 322; Anderson 1997, p. 90; Constantinescu 2012, p. 58). However, there are some philosophers, who do not accept this view. Referring to Chang (2002a) and Gert (2004), Andreou writes: "I am sympathetic with their skepticism about the common assumption that if it is not the case that an option is determinately better than, worse than or equal to another option, then this is necessarily a case of incomparability." (2011, p. 62). According to Chang, "Two items are incomparable if no positive value relation holds between them." (Chang 1997, p. 4). In other words, if no value can be found that allows for comparison, items in hard cases remain incomparable. However, Chang herself has provided an alternative account, rejecting the idea that no other relation but 'better than', 'worse than' and 'equally good to' holds between two items in hard cases. She has argued that two options in hard cases are not incomparable but rather on par with each other, whereby she describes the notion of parity as a nonzero, unbiased difference. (Chang 2002b, p. 168; Chang 1997, p. 26). ${ }^{6}$ Griffin and Parfit have used similar strategies. According to Griffin and Parfit, hard cases should be interpreted as rough comparability (Griffin 1998, p. 96; Parfit 1986, p. 431). They suggest that two items in hard cases are merely in the same league or roughly equal (Parfit 1986, p. 431): If two items are in the same league or roughly equal, then a lack of clarity regarding the items at stake prevents a more precise determination of their relation.

D'Agostino (2003) makes a different suggestion. He maintains that hard cases are incommensurable, whereby this means that a trade-off is not possible. ${ }^{7}$ In standard cases of comparison, agents will trade a certain number of items $x$ (e.g. 5 bananas) for a certain number of items $y$, e.g. 3 apples. Thus, the substitution rate would be 5/3. In cases of incommensurability, so D'Agostino, no such substitution rate exists - the possibility of a substitution rate is crucial for items to be comparable.

While the explication of these notions-incomparability, incommensurability, parity, rough equality - is complex, we can group these various theories into two camps - thereby obviously eliminating both important and subtle distinctions of the accounts at stake: those theories that maintain that in hard cases options are still roughly comparable (Chang, ${ }^{8}$ Griffin, Parfit, Andreou); and those theories that pertain that such a comparison cannot be made (Raz, D'Agostino), i.e. that both options are incomparable.

\footnotetext{
${ }^{6}$ Several philosophers explicate this concept in different ways. See for instance, Gert (2004), Rabinowicz (2008) and Andreou (2015).

7 D'Agostino distinguishes different kinds of incommensurability. We are referring here to the idea, which D'Agostino calls S-Incommensurability (D’Agostino 2003, p. 43).

8 There are important differences between the notions of parity (Chang 2002a, b) and rough equality (Griffin 1997; Parfit 1986). However, as an antipode to strict incomparability, we believe that Chang's, Griffin's and Parfit's theories have more in common than what separates them. For the purposes of this paper, we will therefore treat them under the single heading of theories of rough comparability.
} 
For the purposes of this paper, let us distinguish between strict incomparability and rough comparability. ${ }^{9}$ Whereas strict incomparability maintains that no comparison can be made between options in hard cases, rough comparability holds that a comparison is in principle possible. But how can we decide which of these two positions is correct? In other words, can we operationalize strict incomparability in such a way that we can decide whether two options in hard cases are strictly incomparable or roughly comparable? We believe that such an operationalization is not only possible but, in what follows, we will identify such a mechanism and present an experiment in Sect. 3.3 in which we recorded data that supports one of the interpretations of the small-improvement argument.

The key to operationalizing the phenomenon of strict incomparability is to consider the incentive that is a crucial part in the small-improvement argument. If strict incomparability is true, then no incentive-or value-should exist that makes both options comparable. Applied to our example at hand, no matter how many holidays are added to one option, a career as an artist continues to be (at least to many) neither better nor worse nor equal to a career as a lawyer. In contrast, proponents of the thesis of rough comparability tend to think that if the incentive is appropriate and sufficiently large, people will choose one option over the other, demonstrating that people were comparing both options the whole time. After all, if two options are merely roughly equal, then a nice incentive will make them unequal. A first attempt to adjudicate between both interpretations seems therefore to offer a related and sufficiently high incentive and observe whether people can be convinced to choose either of the two options. However, this will not work. A decision between a career as an artist and a lawyer may be both incomparable but also relatively insignificant when comparing 30 additional holidays with no holidays at all. In such a case, people might make a decision in favour ot the improved option only because they would like to get the incentive. Thus, neither interpretation can be ruled out by showing that most people decide in favor of $x+$ given a large-enough incentive.

Let us therefore go one step further: it makes sense to assume that people have different levels of incentive-thresholds when making a decision in favor of one option, e.g. whereas Steve will decide in favor of a career as a lawyer when two additional holidays are added, Sandra might have to be offered six additional holidays in order to choose a law career. However, these differential responses can also be explained by both interpretations of the small-improvement argument: The strict incomparability theorist will account for these responses by claiming that different people will not attach the same importance to additional holidays. Although both options are incomparable, Steve is convinced by two additional holidays because holidays mean quite a lot to him. Sandra is not so fond of holidays and thus needs to be offered a greater number of holidays in order to be persuaded. In contrast, the rough comparability theorist will explain the differential responses in a different way: The difference between Steve's and Sandra's response is not so much a matter of the importance of being on holidays but it is rather the case that the roughness of the comparability can be larger or smaller. For Steve, the two

\footnotetext{
9 Note that both concepts do not contradict the conclusion of the small-improvement argument.
} 
careers are just about roughly equal. A small incentive is already sufficient to persuade him to choose a career as a lawyer. For Sandra, the comparison between both careers allows for less precision: a larger incentive needs to be offered to her such that she perceives the law career to be more valuable. ${ }^{10}$

If what we discussed in the last paragraph is true, then there is a way to operationalize the phenomenon of strict incomparability. We have claimed that differing responses regarding the perceived thresholds of the incentive in a hard case must be accounted for by the proponent of strict incomparability in a very determinate manner. More specifically, the following hypothesis can be made:

Strict incomparability The threshold value of the incentive in hard cases is negatively correlated with the perceived importance of the incentive.

This operationalization of strict incomparability reflects the need to explain positive decisions in hard cases as decisions in favor of the incentive and not as decisions in favor of one of the original options. If a person attaches great importance to the incentive type (holidays), then a lower magnitude of the incentive (e.g. number of additional holidays) is required to pass the threshold-hence the negative correlation. Proponents of rough comparability, in contrast, will not predict such a negative correlation. The perceived importance of the incentive will determine the value of the roughness only minimally, if at all. Thus, once we know how the perceived importance of the incentive is related to the threshold value of the incentive, we can determine whether strict incomparability or rough comparability provide better interpretations of the conclusion of the small-improvement argument. Crucially, this operationalization can be empirically tested. Before we do so, however, we first need to investigate whether hard cases exist at all.

\section{Empirical part}

We have discussed several scholars who argue that hard cases, in contrast to other cases of comparison, exist and reveal a failure of transitive reasoning. Not everybody has been so optimistic about the existence of hard cases though. Regan (1997) not only believes that comparisons might be possible in all cases, he also emphasizes that "nothing is obvious in this area" (1997, p. 150, our italics). If he is right - and we completely agree with Regan on the latter point-then the most straightforward way to settle this question is to make sure the debate has a solid empirical foundation. More specifically, we think that the intuitive plausibility of many of the claims made in this discussion need to be backed up by empirical data. In our empirical studies, we will focus on three claims in this debate where philosophers largely rely on the intuitive plausibility of exemplary cases:

\footnotetext{
${ }^{10}$ We do not claim that the roughness of hard cases is necessarily independent of the incentive offered. It is likely, however, that this roughness is determined by several factors of which the incentive is only one possible factor.
} 
3.1 hard cases can be distinguished from easy cases,

3.2 failure of transitive reasoning is characteristic for hard cases,

3.3 options in hard cases are incomparable/roughly equal/on par.

In order to investigate claims Sects. 3.1 and 3.2 empirically, we tested people's responses to both easy and hard cases. For Sect. 3.3, we focused on a modified hard case of comparison.

\section{Methods}

All participants were recruited on Amazon's Mechanical Turk and paid a small fee for their participation. In order to examine Sects. 3.1 and 3.2, we conducted two studies. In both studies, participants were randomly assigned to a hard case or to an easy case of comparison.

\section{Study 1:}

- Hard case: career as a lawyer or career as an artist $(\mathrm{N}=99)$

- Easy case: career as a lawyer at Smith and Partners or career as a lawyer at Jones and Partners $(\mathrm{N}=97)$

\section{Study 2:}

- Hard case: going out with friends or doing sports $(\mathrm{N}=105)$

- Easy case: working out at Orange fitness-club or working out at Yellow fitnessclub $(\mathrm{N}=104)$

In all four cases, the subjects were presented with three vignettes and subsequently asked to evaluate the situations they were confronted with. ${ }^{11}$ As an example, here are the three vignettes that we presented in the hard case of Study 1. All other vignettes were equivalent in structure (see "Appendix 2" for the exact wording of the vignettes).

First vignette Imagine you have got the choice between a successful career as a lawyer and a successful career as an artist. How would you evaluate your situation?

a. A career as a lawyer is better than a career as an artist.

b. A career as a lawyer is worse than a career as an artist.

c. A career as a lawyer is neither better nor worse than a career as an artist.

\footnotetext{
${ }^{11}$ The vignettes asked the participants to imagine themselves being faced with the decision at hand, and to evaluate which option they themselves would prefer. This was important, for otherwise we cannot conclude that the option 'neither better nor worse' indicates no preference for either of the two options. According to the Range of Permissible Preference View, people might find 'neither better nor worse' the best answer in decision-making scenarios, because they believe it is permissible for a person to prefer $x$ over $y$ just as it is permissible for a person to prefer $y$ over $x$. The distinction between preference and valuation has been very clearly made by Gert (for a related account based on permissible preference orderings, see Rabinowicz 2008): “(...)preference and valuation are not the same things, and preference is most closely related to choice, while valuation is more closely related to the assessment of choice." (2004, p. 493). In order to avoid that people reason in terms of valuation or permissibility, we did not ask people to tell us which preferences they found permissible. Rather, we requested of them to make a decision based on their own preferences.
} 
Second vignette Now imagine you have got the choice between a successful career as a lawyer plus 2 additional holidays and a successful career as an artist. How would you evaluate your situation?

a. A career as a lawyer plus additional holidays is better than a career as an artist.

b. A career as a lawyer plus additional holidays is worse than a career as an artist.

c. A career as a lawyer plus additional holidays is neither better nor worse than a career as an artist.

Third vignette Now, lastly, imagine you have got the choice between a successful career as a lawyer plus 2 additional holidays and a successful career as a lawyer without 2 additional holidays. How would you evaluate your situation?

a. A career as a lawyer plus additional holidays is better than a career as a lawyer.

b. A career as a lawyer plus additional holidays is worse than a career as a lawyer.

c. A career as a lawyer plus additional holidays is neither better nor worse than a career as a lawyer.

\subsection{Hard cases versus easy cases}

In the previous section we have argued that the test bed for the distinction between hard and easy cases are the first two premises of the small-improvement argument. In hard cases, a majority of people who endorse (1) should also endorse (2):

1. $x$ is neither better nor worse than $y$

2. $x+$ is neither better nor worse than $y$

In contrast, in an easy case, those participants who agree to (1) should not endorse (2) but instead hold that ' $x+$ is better than $y$ '.

\section{Results}

To assess the impact of easy versus hard cases on people's decisions, we analyzed whether the responses from all participants who chose 'neither better nor worse' in the first vignettes of Study 1 and 2, would show different response profiles for easy and hard cases in the second vignettes. In Study 1, only $9.8 \%(\mathrm{~N}=9)$ of the participants who selected 'neither better nor worse' in the first vignette of the easy choice condition, also selected 'neither better nor worse' when a small incentive was added (additional holidays). $84.7 \%(\mathrm{~N}=78)$ of the participants chose a career at the law firm which also provided two additional holidays. In contrast, in the hard case condition, $78.6 \%(\mathrm{~N}=33)$ of the participants who selected 'neither better nor worse' in the first vignette, also selected the response 'neither better nor worse' when a small incentive was added. Merely $21.4 \%(\mathrm{~N}=9)$ of the participants chose a career as a lawyer over a career as an artist when two additional holidays were 
offered. A Pearson's Chi-Square Test revealed that the difference between ratings in the easy versus the hard case was highly significant: $\chi^{2}=63.41, p<0.001$, twotailed. Similar results obtained in Study 2: 41 participants did not prefer either the 'doing sports' or the 'going out with friends' option in the hard case scenario. $60.0 \%$ of those subjects did not favor any of the two options when a small incentive was added. In the easy case, a large majority $(77.4 \%)$ of the participants decided in favor of one fitness club once a small incentive was presented. The difference between the easy and hard case conditions was significant: $\chi^{2}=20.34, p<0.01$, two-tailed. The percentages of the responses of both studies are also shown in Fig. 1.

\section{Discussion}

The results clearly show that people's response patterns are different for easy and hard cases of comparison. Easy cases are characterized by two seemingly equal options. Once an incentive is presented, a large majority favor one option over the other-indicating that the original choice was between equally good options. Hard cases do not show the same pattern of responses: The majority of participants who favor neither option in the original scenarios (lawyer vs. artist and going out with friends vs. doing sports) are not persuaded by an additional incentive, even if the incentive consists of two additional holidays (respectively one dollar), demonstrating that the original options were not considered to be equally good. Nonetheless, quite a large number of people in hard cases did consider the improved option $x+$ to be better than the alternative option $(y)$. How can we explain this outcome? First, it might well have been the case that other participants thought of both careers as equally desirable. For those participants, the choice did not present a hard case of comparison. Second, the two additional holidays that we presented in the second vignettes might have been already too tempting for those people who value holidays very much. They therefore decided in favor of the improved option although the original case was indeed a hard case of comparison. In Sect. 3.3 we deal with this possibility in greater detail.

The results show different response patterns for easy and hard cases of comparison and in turn suggest that hard cases are real-world phenomena that are different from easy cases of comparison. However, the epistemic limitations view provides an alternative and hence competing explanation of the results of the career choice experiment. ${ }^{12}$ Two possible versions of this view can be distinguished. In a decision such as choosing between different careers, an agent is either faced with uncertainty about matters of non-normative facts or with uncertainty about matters of normative facts. The former case can be analyzed within the framework of decision theory. Accordingly, an agent is faced with a decision under risk or a decision under ignorance (see for instance Resnik 1990 or De Jonge 2012). Most choices are made against a background of various states of affairs. Depending on what states will come true, different outputs are realized and since the agent can-if at all-only assign probabilities to these states, there is a high degree of factual

\footnotetext{
${ }^{12}$ We thank a reviewer for this journal for suggestions regarding the epistemic limitations view.
} 


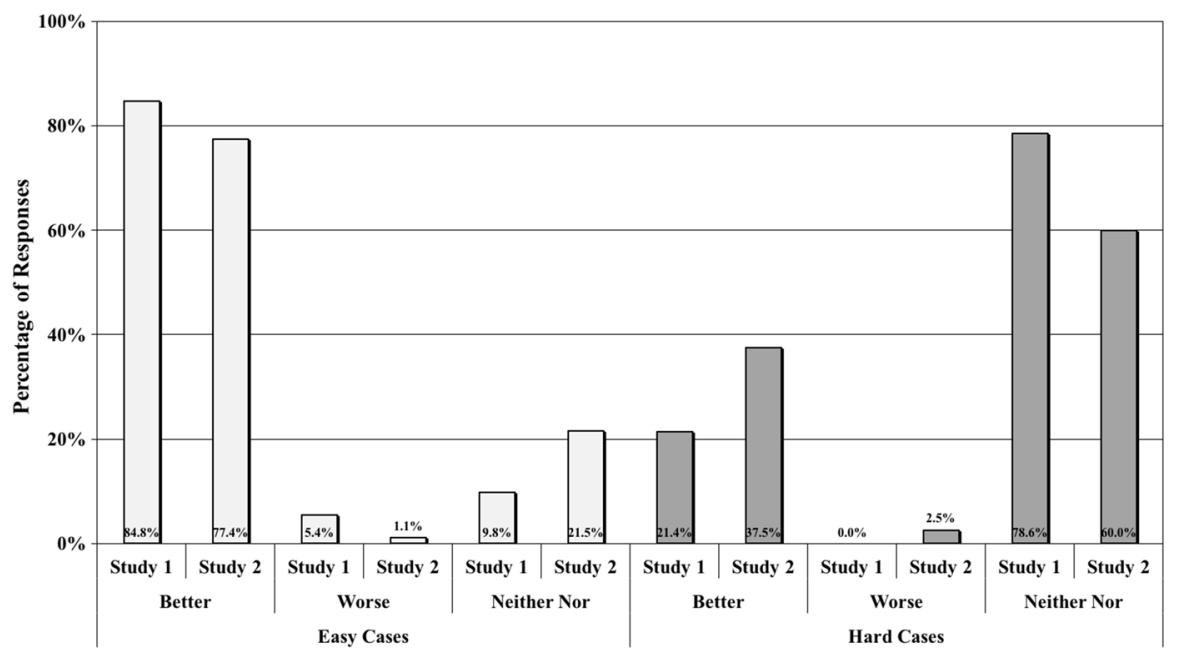

Fig. 1 Responses to the second vignettes. On the left (in light grey): a majority of people in easy cases prefer the option to which a small incentive is added. On the right (in dark grey): in hard cases, most people opt for the 'neither better nor worse' option despite the fact that an incentive was added to the first option

uncertainty, or as Jensen puts it: "Almost any decision one can think of is taken in a context in which the decision maker has only limited information." (Jensen 2011, p. 406). Now, observe that the hard case version of the career scenario presumably has a relatively high degree of factual uncertainty compared to the easy case version: it is harder to predict the possible outcomes of pursuing a law or art career than predicting the outcome of pursuing two law careers in New York. If this is correct then differences in the responses between easy and hard cases might be due to the uncertainty of matters of non-normative facts. However, the vignettes of Study 2 (going out with friends vs. doing sports) do not contain the same asymmetry in the degree of uncertainty between the easy and hard cases of comparison. Most people know pretty well the possible outcomes of going out with friends and doing sports. ${ }^{13}$ Given that the response patterns did not differ significantly between Study 1 and 2, the first version of the epistemic limitations view seems not very suitable to provide an alternative explanation of our results. The second version of the epistemic limitations view concerns normative uncertainty about the outputs, i.e. uncertainty about one's own preferences (see e.g. Sepielli 2009). Accordingly, more people select 'neither better nor worse' in hard case scenarios not because people have no preference for either option but because both options are too close to call, i.e. the difference between one's preferences for the two options is epistemically opaque. However, while this account sounds convincing for a single choice, it seems less

\footnotetext{
13 Related to this, we follow Chang (2002a, b, 2012) who argues against the epistemic limitations view. Chang holds the position that in hard cases of comparison we often have enough practical certainty to be sure that one option is neither better, nor worse, nor equal to another option, i.e. that people know which factors matter for the decision.
} 
reasonable when considering the small incentive option. In the second vignettes, an incentive is added to one option. If there were indeed a fine-grained epistemic limitation, it seems implausible that a majority of the participants continue to fail to introspect their preferences when being confronted with the second choice.

\subsection{Failure of transitivity}

The results presented in Sects. 3.1 show that a distinction between easy and hard cases is indeed well motivated and empirically supported. However, most philosophers do not focus on distinguishing both cases of comparison, they make specific claims about hard cases. Most importantly, they argue that people violate transitvity. If failure of transitivity is indeed characteristic of hard cases, we can predict that people who endorse (1) and (2) should also endorse (3):

1. $x$ is neither better nor worse than $y$,

2. $x+$ is neither better nor worse than $y$,

3. $x+$ is better than $x$.

\section{Results}

In order to investigate whether failure of transitive reasoning is characteristic for hard cases, we analyzed how many people actually violated transitivity in both studies. In Study 1, of all those participants who responded with "neither better nor worse' to the first two vignettes, $60.6 \%(\mathrm{~N}=20)$ indeed violated the transitivity requirement. This was, however, not significantly above chance level, $\chi^{2}=2.25, p$ $=0.134$, two-tailed. In the easy case condition, only a single person violated the transitivity requirement. In Study 2, a greater number of participants failed the transitivity condition $(70.8 \%)$. This result was significantly above chance:

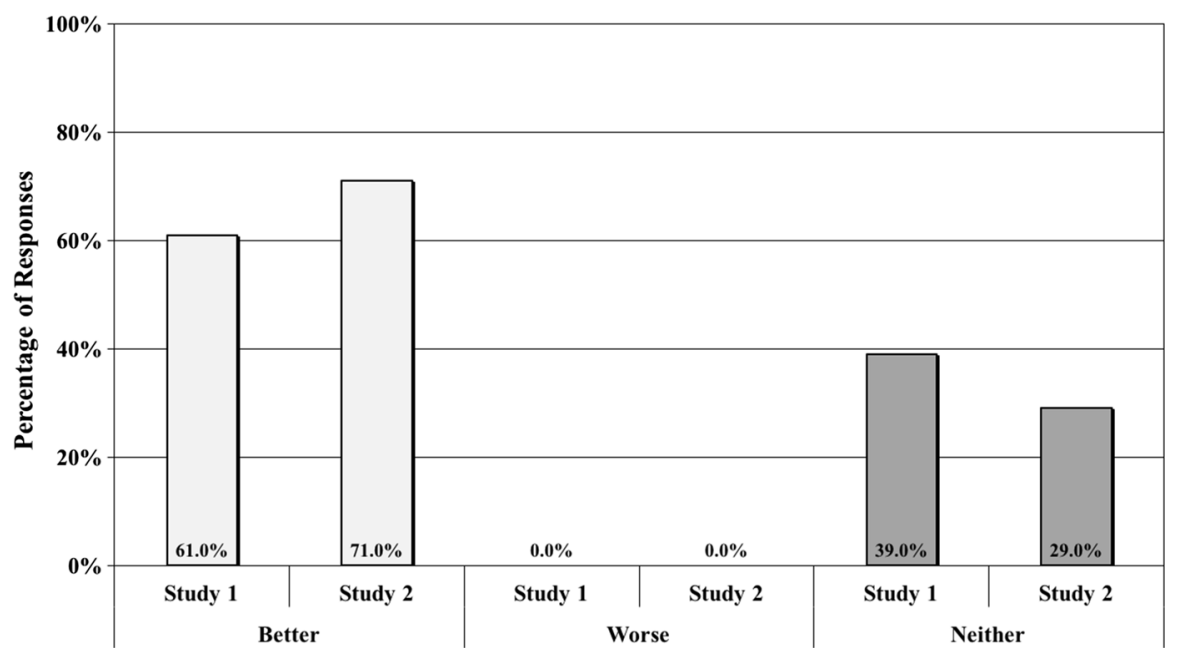

Fig. 2 Participant's responses to the third vignettes, assessing the claim that in hard cases transitivity is violated. In both studies, a majority of the participants violated transitivity (light bars on the left), whereas a smaller percentage did not fail the transitivity condition 
$\chi^{2}=9.32, p=0.002$, two-tailed. Four participants, i.e. $20 \%$ violated transitivity in the easy condition of Study 2. The percentages in response to the third vignettes are depicted in Fig. 2.

\section{Discussion}

The analysis of people's responses to the third vignettes brought to light that a majority of the participants violated transitivity. While this outcome was significant in Study 2, a substantial minority did not fail the transitivity requirement, especially in Study 1. Three explanations might be given to account for this effect. First, some participants might have been aware that a different response would have violated transitivity and hence decided to opt for a less plausible but consistent (in the sense of transitivity) response. Second, having responded twice before (first and second vignette) with 'neither better nor worse', some participants might have chosen the same response either due to lack of attention or lack of motivation. Third, those people who did not fail the transitivity condition, might have considered the incentive as too small to be bothered to favor the option $x+.{ }^{14}$

\subsection{Do we think of options in hard cases as incomparable?}

We have seen that different philosophers argue for different interpretations of the conclusion of the small-improvement argument. While some, e.g., Griffin (1997), Parfit (1986) and Andreou (2011) interpret options in hard cases of comparison as being roughly comparable, others (Raz 1986; D'Agostino 2003) believe that the options in hard cases are incomparable. In (2.3) we have suggested a way to operationalize the notion of strict incomparability. By relating the perceived importance of the incentive - that is added to one of the options in hard cases- to the threshold value of the incentive, we can determine whether or not any correlation exists between those variables. Focusing on incomparability, the following hypothesis has been developed in (2.3).

Strict incomparability The threshold value of the incentive in hard cases is negatively correlated with the perceived importance of the incentive.

\section{Methods}

Artists tend to be less constrained in terms of holidays than lawyers. In Study 3, we therefore decided to present participants $(\mathrm{N}=155)$ with a variation of vignette 1 from Study 1 . The only difference consisted in asking participants to evaluate the situation between having a successful career as a lawyer and a successful career as an architect. Those participants $(\mathrm{N}=71)$ who responded that a career as a lawyer was neither better nor worse than a career as an architect, were then randomly assigned to one of the following two conditions:

\footnotetext{
14 In a follow-up study (Reuter and Messerli 2016) we investigate the impact of the importance of a decision on violations of transitvity.
} 
Condition 1: Becoming a lawyer $(\mathrm{N}=36)$

You have decided that a successful career as a lawyer is neither better nor worse than a successful career as an architect. How many additional holidays would a law firm need to offer so that you would decide in favor of a career as a lawyer compared to a career as an architect?

Condition 2: Becoming an architect $(\mathrm{N}=35)$

You have decided that a successful career as a lawyer is neither better nor worse than a successful career as an architect. How many additional holidays would an architecture firm need to offer so that you would decide in favor of a career as an architect compared to a career as a lawyer?

Participants answered on a scale from 0 additional holidays to 21 additional holidays. The third question of Study 3 asked people the following question:

How important do you consider holidays to be for you?

Responses were recorded on a 21-point Likert scale anchored at ' -10 ' meaning 'not at all important', '0' meaning 'somewhat important' and '+10' meaning 'very important'.

\section{Results}

The average amount of holidays that the 73 participants wanted to be offered to decide in favor of either a career as a lawyer or a career as an architect was 9.14 days ( $\mathrm{SD}=6.28$ ). The mean value for those participants assigned to the (Becoming a lawyer) question was 11.42 (SD = 6.59) and for (Becoming an architect) was 7.03 ( $\mathrm{SD}=5.15$ ). As regards to the importance of holidays, the average value was 4.04 ( $\mathrm{SD}=5.32$ ). In order to test the hypothesis stated above, we carried out a simple linear regression to predict the number of additional holidays based on people's ratings of the importance of holidays. The analysis yielded a Pearson's correlation coefficient of $r=-0.07$. No significant correlation was found, $\mathrm{F}(1,69)=0.29, p=0.586$. Due to the difference in the average rating of number of holidays in both conditions, we conducted linear regressions for each condition. No significant correlations were found; Becoming a lawyer: $\mathrm{F}(1,34)=$ $0.29, p=0.866$; Becoming an architect: $\mathrm{F}(1,33)=0.22, p=0.643$. The data points as well as the regression line are depicted in Fig. 3.

\section{Discussion}

The results of the simple linear regression revealed no significant correlation between additional holidays and perceived importance of holidays. While we cannot conclude that no correlation exists, given the null hypothesis, the data strongly suggests that people's perception of the importance of holidays does not predict and hence influence the number of additional holidays needed to choose one career over the other. Only $0.4 \%$ of the variability in the amount of holidays could be accounted for by the importance of the holidays. As we have outlined above, this result aligns with the predictions of the rough comparability view and goes against the strict incomparability account. 


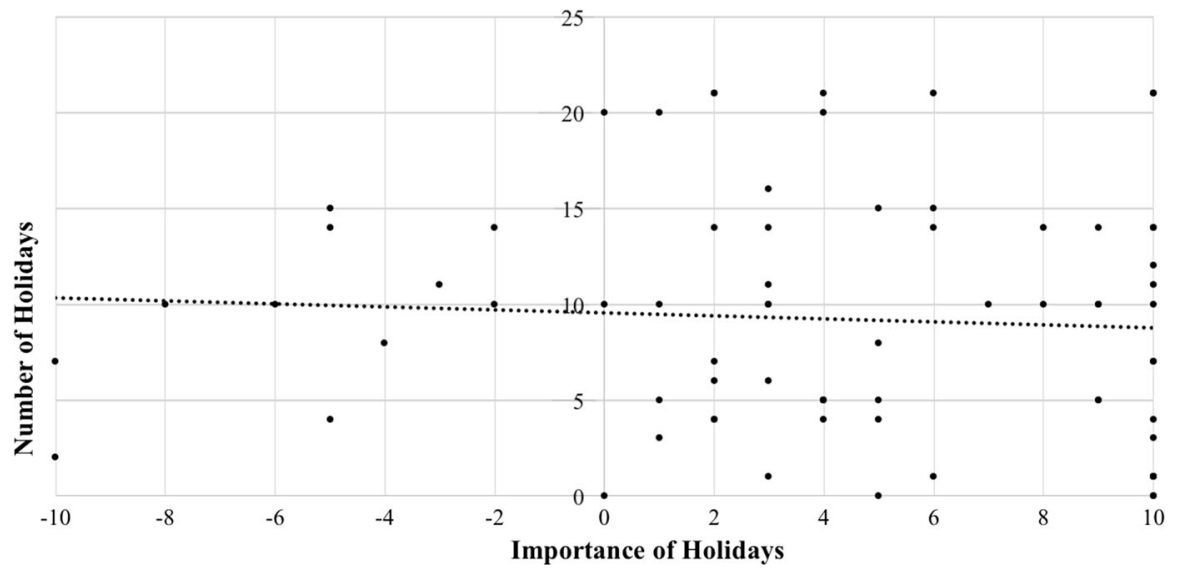

Fig. 3 Data points relating the importance of holidays with the threshold-value of holidays. The line indicates the correlation function between both variables

\section{General discussion}

For the last 30 years, philosophers have discussed hard cases of comparison in rational decision-making processes, as well as different ways of characterizing these hard cases. Within these debates, it is usually assumed that hard cases indeed exist (and as such present real-world dilemmas). Moreover, a large consensus has evolved, claiming that hard cases imply intransitive reasoning. So far, both the assumption and the consensus are merely supported by the intuitive plausibility of exemplary cases, whereby the intuitive plausibility is judged by experts in the field. Recently, however, the experimental philosophy movement has put severe pressure on examining expert intuitions, for too often expert intuitions neither reflect the intuitions of lay people, nor do they function as stable and robust pieces of evidence (Machery et al. 2004; Swain et al. 2008). A similar charge can be mounted against the discussion at hand: If lay people do not distinguish easy from hard cases, and / or do not violate transitivity when encountering hard cases, the debate becomes almost pointless-merely relevant for the reasoning processes of the very few.

We therefore started to investigate the claims of this debate empirically. Fortunately for the philosophical consensus - but maybe less interesting to somewe were able to confirm the reliability and representative character of the expert's intuitions in regards to two central assertions: First, the results of our experiments clearly show that a large majority of people distinguish hard cases from easy ones and reveal response patterns that are predicted by the philosophical consensus. Second, a substantial majority of people violate transitivity when reasoning about hard cases. Having used two different studies, i.e. decisions in regard to career choice and leisure time, our results do not seem to depend on either the specific choice a person is confronted with or the exact wording of the questions. Instead, it is likely that our results are robust and easily replicable. While the philosophical consensus has predicted such an outcome, some philosophers have argued against 
the mainstream. Regan (1997) has not only expressed a reasonable skepticism about the obviousness of the consensus - which we appreciate- he has also argued that hard cases might not exist (or are at least not as widespread as one might think). In other words, Regan has argued in favor of strong comparability even in the examples we discussed above. However, Regan's position seems to be refuted by our empirical data.

Not only have we empirically investigated the philosophical consensus, we also aimed to operationalize one of the main interpretations of the conclusion of the small-improvement argument and to provide empirical results that can move this debate forward. In this process of operationalization we had to disregard important details of many theories on hard cases of comparison. Nonetheless, even a fairly crude operationalization is probably better than none, even if it merely helps to distance oneself from one specific interpretation. In a nutshell, the idea was to use different predictions that various theories would make about the nature of incentives in decision-making processes. Whereas strict incomparabilitists think that no incentive will be strong enough to make the original options comparable, advocates of rough comparability think otherwise. Our results from Sect. 3.3 support a reading of hard cases according to which two options are not incomparable in a strict sense but actually roughly comparable. More specifically, our data shows that the perceived importance of the incentive does not correlate with the magnitude of the incentive. This correlation should have been observed if strict incomparability is the case. Theories of rough equality and parity are consistent with the fact that no such correlation was found. Thus, our investigation favors a specific interpretation of hard cases, i.e. that two options that are neither better, nor worse, nor equally good, can nevertheless be comparable. We do not, of course, have shown that no hard cases exist in which two options are conceived of as truly incomparable. This study has only investigated a single hard case. Thus, it is quite possible that hard cases fall into two sets: those in which options are roughly equal and those in which the options are incomparable. Where does all that leave us?

We have begun our discussion with an argument linking rational choice with hard cases of comparison:

i. The possibility of rational choice between two options depends on them being comparable.

ii. Two options, $x$ and $y$, are comparable, if and only if $x$ is either better than, worse than, or equal to $y$.

iii. There are hard cases, in which $x$ is neither better, nor worse, nor equally good to $y$.

$\therefore$ There are hard cases, in which a rational decision cannot be made.

In this paper, we have examined the truth of premises (ii) and (iii) of the above argument. We have provided empirical evidence in favor of premise (iii): many people agree with all three premises of the small-improvement argument, and hence it seems we can frame hard cases as choices in which one option is neither better than, nor worse than, nor equally good to another option. However, and importantly, our results also show that premise (ii) does not seem to be the case. Although one 
option might be neither better, nor worse, nor equally good to another option, people seem to compare these options. Once an incentive is provided that exeeds a certain individual threshold, people decide in favor of the improved option. This is not due to the fact that the incentive becomes the center of the decision making process, but because the area of rough equality has been 'left behind'.

So, are people rational even in hard cases of comparison? This all depends on which aspect of rationality is emphasized. According to the argument above and the data we provided against premise (ii), hard choices are rational decisions. However, we should not forget that people violate transitivity in hard cases. Thus, if transitive reasoning is at the heart of rationality, people do in fact show at least some form of irrationality in hard cases of comparison. This discussion, however, would leave the purely descriptive realm that we have intended to tackle in this paper.

\section{Conclusion}

Hard cases of comparison are a fascinating test bed for rational-decision making processes that have been at the center of philosophical debates for almost three decades. Our aim in this paper was to (a) provide an empirical foundation to advance these debate, as well as (b) investigate empirical avenues for the operationalization of various interpretations. Our studies have shown that the experts' intuitions in this particular field are spot on in highlighting the special status of hard cases and characterizing such cases as instances in which agents violate transitivity. We have also suggested one particular way to adjudicate between various interpretations of the small-improvement argument. If our method is sound and our data robust, the results put pressure on advocates of incomparability: People seem to be capable to compare both options in at least some hard cases of comparison.

Acknowledgments We would like to thank Claus Beisbart, Monika Betzler, Georg Brun, Hans Rott, Stephan Sellmaier, and Jan Walker for their support and comments on previous drafts of the article. The paper was presented at conferences in Bern and Reading. We thank the audiences for their valuable feedback.

\section{Appendix 1: The role of (in)transitivity in the small-improvement argument}

In part 2 of the paper we have claimed that the role of (in)transitivity within the small-improvement argument is more complex, i.e. that some versions of the argument also involve a different form of transitivity. Let us first restate the premises of the small-improvement argument:

$$
\begin{array}{lll}
1 & \neg(x \succ y) \wedge \neg(y \succ x) & \text { premise } \\
2 & \neg(x+\succ y) \wedge \neg(y \succ x+) & \text { premise } \\
3 & x+\succ x & \text { premise }
\end{array}
$$


The crucial point regarding the role of transitivity within the argument is contained in the inference from premises (2) and (3) from which it is concluded that both options are not equally good. In order to validate this conclusion, additional assumptions are needed. The following formalization shows that the smallimprovement argument (SIA) requires transitivity:

$$
\begin{array}{lll}
1 & \neg(x+\succ y) & \text { premise } 2 \text { of the SIA } \\
2 & x+\succ x & \text { premise } 3 \text { of the SIA } \\
3 & x+\succ x \wedge x=y \rightarrow x+\succ y & \text { transitivity } \\
4 & \neg(x+\succ x \wedge x=y) & 1,3 \text { MTT } \\
\text { C } & {[\therefore] \neg(x=y)} & 2,4 \text { MPT }
\end{array}
$$

According to the formalizations given above, the following transitivity-condition is required as a premise in order to arrive at the conclusion:

$$
x+\succ x \wedge x=y \rightarrow x+\succ y
$$

Espinoza, following Savage, calls this equation 'indifference principle' (Savage 1954, p. 17; Espinoza 2008, p. 130.), while Gustafsson and Espinoza call it PItransitivity (Gustafsson and Espinoza 2010, p. 755). This principle of transitivity can be interpreted either as normative assumption or as an empirical hypothesis. As a normative assumption this principle states that if two options are valued equally, then a small improvement on either option requires the agent to prefer the improved one. As an empirical hypothesis this principle states that if two options are valued equally, a small improvement on either option makes the agent preferring the improved one. Gustafsson and Espinoza (2010) therefore claim that transitivity is one of the core premises of the small-improvement argument.

However, this claim can be challenged, since there are alternative formalizations which do not involve transitivity. The following formalization, for instance, does not involve transitivity:

$$
\begin{array}{lll}
1 & \neg(x+\succ y) & \text { premise } 2 \text { of the SIA } \\
2 & x+\succ x & \text { premise } 3 \text { of the SIA } \\
3 & x=y & \text { premise } \\
4 & \neg(x+\succ x) & 1,3 x=y \\
5 & x+\succ x \wedge \neg(x+\succ x) & 2,4 \wedge I \\
\text { C } & {[\therefore] \neg(x=y)} & 3,5 \text { reductio }
\end{array}
$$

Assuming first that $x$ and $y$ are equally good, we then deduced a contradictionsubstituting $x$ and $y$ in premise (1). This leads to a reductio of premise (3). The opposite of the assumed premise must be true, i.e. $x$ and $y$ are not equally good.

Thus, the premises of the small-improvement argument involve intransitivitywe have shown that in the paper, but the conclusion of the argument, i.e. that one option is neither better, nor worse, nor equally good to another option, may also assume a different kind of transitivity. 


\section{Appendix 2: Vignettes of Study 1 and Study 2}

\section{Study 1: Hard case}

First vignette Imagine you have got the choice between a successful career as a lawyer and a successful career as an artist. How would you evaluate your situation?

- A career as a lawyer is better than a career as an artist.

- A career as a lawyer is worse than a career as an artist.

- A career as a lawyer is neither better nor worse than a career as an artist.

Second vignette Now imagine you have got the choice between a successful career as a lawyer plus 2 additional holidays and a successful career as an artist. How would you evaluate your situation?

- A career as a lawyer plus additional holidays is better than a career as an artist.

- A career as a lawyer plus additional holidays is worse than a career as an artist.

- A career as a lawyer plus additional holidays is neither better nor worse than a career as an artist.

Third vignette Now, lastly, imagine you have got the choice between a successful career as a lawyer plus 2 additional holidays and a successful career as a lawyer without 2 additional holidays. How would you evaluate your situation?

- A career as a lawyer plus additional holidays is better than a career as a lawyer.

- A career as a lawyer plus additional holidays is worse than a career as a lawyer.

- A career as a lawyer plus additional holidays is neither better nor worse than a career as a lawyer.

\section{Study 1: Easy case}

First vignette Smith and Partners as well as Jones and Partners are two successful law firms in New York. Now imagine you have got the choice between a successful career as a lawyer at Smith and Partners and a successful career as a lawyer a Jones and Partners. How would you evaluate your situation?

- A career as a lawyer at Smith and Partners is better than a career as a lawyer at Jones and Partners.

- A career as a lawyer at Smith and Partners is worse than a career as a lawyer at Jones and Partners.

- A career as a lawyer at Smith and Partners is neither better nor worse than a career as a lawyer at Jones and Partners. 
Second vignette Now imagine you have got the choice between a successful career as a lawyer at Smith and Partners plus 2 additional holidays and a successful career as a lawyer at Jones and Partners without additional holidays. How would you evaluate your situation?

- A career as a lawyer at Smith and Partners plus additional holidays is better than a career as a lawyer at Jones and Partners.

- A career as a lawyer at Smith and Partners plus additional holidays is worse than a career as a lawyer at Jones and Partners.

- A career as a lawyer at Smith and Partners plus additional holidays is neither better nor worse than a career as a lawyer at Jones and Partners.

Third vignette Now, lastly, imagine you have got the choice between a successful career as a lawyer plus 2 additional holidays and a successful career as a lawyer without 2 additional holidays. How would you evaluate your situation?

- A career as a lawyer plus additional holidays is better than a career as a lawyer.

- A career as a lawyer plus additional holidays is worse than a career as a lawyer.

- A career as a lawyer plus additional holidays is neither better nor worse than a career as a lawyer.

\section{Study 2: Hard case}

First vignette Imagine you have got the choice between two different ways of spending your spare time: You can either go out with friends once a week or you can do sports once a week. How would you evaluate your situation?

- Going out with friends once a week is better than doing sports once a week.

- Going out with friends once a week is worse than doing sports once a week.

- Going out with friends once a week is neither better nor worse than doing sports once a week.

Second vignette Now imagine you have got the choice between the following options. You can either go out with friends once a week plus you get one dollar or you can do sports once a week. How would you evaluate your situation?

- Going out with friends once a week plus an extra dollar is better than doing sports once a week.

- Going out with friends once a week plus an extra dollar is worse than doing sports once a week.

- Going out with friends once a week plus an extra dollar is neither better nor worse than doing sports once a week. 
Third vignette Now, lastly, imagine you have got the choice between the following options: You can either go out with friends once a week plus you get one dollar or you can go out with friends once a week. How would you evaluate your situation?

- Going out with friends once a week plus an extra dollar is better than going out with friends.

- Going out with friends once a week plus an extra dollar is worse than going out with friends.

- Going out with friends once a week plus an extra dollar is neither better nor worse than going out with friends.

\section{Study 2: Easy case}

First vignette Orange Fitness Club as well as Yellow Fitness Club are two great fitness clubs in New York. Now imagine you have got the choice between two ways of spending your spare time: You can either do your weekly workout at Orange Fitness Club or you can do your weekly workout at Yellow Fitness Club. How would you evaluate your situation?

- A weekly workout at Orange Fitness Club is better than a weekly workout at Yellow Fitness Club.

- A weekly workout at Orange Fitness Club is worse than a weekly workout at Yellow Fitness Club.

- A weekly workout at Orange Fitness Club is neither better nor worse than a weekly workout at Yellow Fitness Club.

Second vignette Now imagine you have got the choice between the following options: You can either do your weekly workout at Orange Fitness Club plus you get one dollar or you can do your weekly workout at Yellow Fitness Club. How would you evaluate your situation?

- A weekly workout at Orange Fitness Club plus an extra dollar is better than a weekly workout at Yellow Fitness Club.

- A weekly workout at Orange Fitness Club plus an extra dollar is worse than a weekly workout at Yellow Fitness Club.

- A weekly workout at Orange Fitness Club plus an extra dollar is neither better nor worse than a weekly workout at Yellow Fitness Club.

Third vignette Now, lastly, imagine you have got the choice between the following options: You can either do your weekly workou at Orange Fitness Club plus you get one dollar or you can do your weekly workout at Orange Fitness Club. How would you evaluate your situation? 
- A weekly workout at Orange Fitness Club plus an extra dollar is better than a weekly workout at Orange Fitness Club.

- A weekly workout at Orange Fitness Club plus an extra dollar is worse than a weekly workout at Orange Fitness Club.

- A weekly workout at Orange Fitness Club plus an extra dollar is neither better nor worse than a weekly workout at Orange Fitness Club.

\section{References}

Anderson, E. (1997). Practical reason and incommensurable goods. In R. Chang (Ed.), Incommensurability, incomparability, and practical reason (pp. 90-109). Cambridge: Harvard University Press.

Andreou, C. (2011). Choosing well: Value pluralism and patterns of choice. In T. Brooks (Ed.), New waves in ethics (pp. 48-63). Basingstoke: Palgrave Macmillan.

Andreou, C. (2015). Parity, comparability, and choice. Journal of Philosophy, 112(1), 5-22.

Boot, M. (2007). Incommensurability, Incomplete Comparability and the Scales of Justice. Dissertation, University of Oxford.

Boot, M. (2009). Parity, incomparability and rationally justified choice. Philosophical Studies, 146(1), 75-92.

Bridges, D., \& Mehta, G. (2013). Representations of preferences orderings. Berlin: Springer.

Carlson, E. (2011). The small-improvement argument rescued. The Philosophical Quarterly, 61(242), $171-174$.

Chang, R. (1997). Introduction. In R. Chang (Ed.), Incommensurability, incomparability, and practical reason (pp. 1-34). Cambridge: Harvard University Press.

Chang, R. (2002a). The possibility of parity. Ethics, 112(4), 659-688.

Chang, R. (2002b). Making comparisons count. New York: Routledge.

Chang, R. (2012). Are hard choices cases of incomparability? Philosophical Issues, 22(1), 106-126.

Chang, R. (2014). The existentialist of hard choices. (Interview by Richard Marshall with Ruth Chang). 3: AM Magazine. http://www.3ammagazine.com/3am/the-existentialist-of-hard-choices/.

Constantinescu, C. (2012). Value incomparability and indeterminacy. Ethical Theory and Moral Practice, 15(1), 57-70.

D'Agostino, F. (2003). Incommensurability and commensuration. Hampshire: Ashgate.

De Jonge, J. (2012). Rethinking rational choice theory. Basingstoke: Palgrave Macmillan.

De Sousa, R. (1974). The good and the true. Mind, 83(332), 534-551.

Espinoza, N. (2008). The small improvement argument. Synthese, 165(1), 127-139.

Gert, J. (2004). Value and parity. Ethics, 114(3), 492-510.

Griffin, J. (1997). Incommensurability: What's the problem? In R. Chang (Ed.), Incommensurability, incomparability, and practical reason (pp. 35-51). Cambridge: Harvard University Press.

Griffin, J. (1998). Value judgement: Improving our ethical beliefs. Oxford University Press.

Gustafsson, J. (2013). Indeterminacy and the small-improvement argument. Utilitas, 25(4), 433-445.

Gustafsson, J., \& Espinoza, N. (2010). Conflicting reasons in the small-improvement argument. The Philosophical Quarterly, 60(241), 754-763.

Hsieh, N. (2005). Equality, clumpiness and incomparability. Utilitas, 17(2), 180-204.

Jensen, K. (2011). A philosophical assessment of decision theory. In S. Röser (Ed.), Handbook of risk theory (pp. 405-439). Berlin: Springer.

Machery, E., Mallon, R., Nichols, S., \& Stich, S. P. (2004). Semantics, cross-cultural style. Cognition, 92(3), 1-12.

Parfit, D. (1986). Reasons and persons. Oxford: Clarendon Press.

Raz, J. (1986). The morality of freedom. Oxford: Clarendon Press.

Rabinowicz, W. (2008). Value relations. Theoria, 74(1), 18-49. 
Regan, D. (1997). Value, comparability, and choice. In R. Chang (Ed.), Incommensurability, incomparability, and practical reason (pp. 129-150). Cambridge: Harvard University Press.

Resnik, M. (1990). Choices an introduction to decision theory. Minneapolis: University of Minnesota Press.

Reuter, K., \& Messerli, M. (2016). How not to characterize a hard choice (Unpublished manuscript).

Savage, L. (1954). The foundations of statistics. New York: Wiley.

Schaber, P. (1994). Sind alle Werte vergleichbar? Kosten-Nutzen-Analyse und das Inkommensurabilitätsproblem. Analyse und Kritik, 16(2), 153-165.

Sepielli, A. (2009). What to do when you don't know what to do. Oxford studies in metaethics, 4, 5-28.

Swain, S., Alexander, J., \& Weinberg, J. M. (2008). The instability of philosophical intuitions: Running hot and cold on truetemp. Philosophy and phenomenological research, 76(1), 138-155. 